\title{
The effect of synbiotics supplement on alcohol use disorders identification test and biochemical parameters, gamma glutamyl transferase, lipopolysaccharide and immunoglobulin a levels, in high risk alcoholics
}

\author{
Vachrintr Sirisapsombat ${ }^{1}$, Parama Pratummas ${ }^{2}$, Phuttharaksa Phumcharoen ${ }^{3}$, \\ Thamthiwat Nararatwanchai ${ }^{4}$, Chaiyavat Chaiyasut ${ }^{5}$, Sasithorn Sirilun ${ }^{6}$, \\ Phakkharawat Sittiprapaporn ${ }^{7}$ \\ 1,2,3 Doctoral Student, Brain Science and Engineering Innovation Research Group, School of Anti-Aging and \\ Regenerative Medicine, Mae Fah Luang University, Bangkok, Thailand, and Department of Anti-Aging Medicine, \\ School of Anti-Aging and Regenerative Medicine, Mae Fah Luang University, Bangkok, Thailand, ${ }^{4}$ Professor, \\ Department of Anti-Aging Medicine, School of Anti-Aging and Regenerative Medicine, Mae Fah Luang University, \\ Bangkok, Thailand, ${ }^{5}$ Assistant Professor and Head, ${ }^{6}$ Assistant Professor, Innovation Center for Holistic Health, \\ Nutraceuticals and Cosmeceuticals, Faculty of Pharmacy, Chiang Mai University, Chiang Mai, Thailand, ${ }^{7}$ Assistant \\ Professor and Head, Brain Science and Engineering Innovation Research Group, School of Anti-Aging and \\ Regenerative Medicine, Mae Fah Luang University, Bangkok, Thailand, and Department of Anti-Aging Science, School \\ of Anti-Aging and Regenerative Medicine, Mae Fah Luang University, Bangkok, Thailand
}

Background: Alcohol consumption does not result in dependence or abuse among most people. Nevertheless, a significant group of the population as a whole unavoidably is troubled by chronic alcoholism. Alcohol is involved in a number of diseases, disorders, and injuries, and several social problems. Aims and Objective: To investigate the possible effects of synbiotics supplement affecting to gut-brain axis in high risk alcohol drinkers through alterations between improving of gut related parameters and changes of alcohol use disorders identification test (AUDIT). Materials and Methods: Single group, pre- and post-test study. Participants: 24 male participants, alcohol use disorders identification test at 8 or above. Exclusions included clinical diagnosis of cirrhosis, immunodeficiency, autoimmune disorder, use of drugs other than alcohol, pregnancy and lactation, use of antibiotics and herbs during the course of study. Intervention: Synbiotics containing probiotics 7 species and prebiotic 3 types once a day before bedtime for 8 weeks. Main outcome measures: Primary outcome- the efficacy of synbiotics supplement improving subjective AUDIT score. Secondary outcome- changes on gut related biochemical parameters (gamma glutamyl transferase, lipopolysaccharide and immunoglobulin A levels). Results: Twenty high risk alcoholic participants (with an average age of $42.50 \pm 11.66$ years) were supplemented with synbiotics contained 6.25 billion cells of probiotics per day for 8 weeks. After the end of intervention, there was significantly improved total AUDIT score $(p=0.001)$. The changes in gamma glutamyl transferase (GGT), lipopolysaccharide and immunoglobulin A level was calculated. GGT (from 90.62 \pm 56.65 $\mathrm{U} / \mathrm{l}$ to $67.67 \pm 57.00 \mathrm{U} / \mathrm{l}$ ), lipopolysaccharide (from $23.19 \pm 9.57$ to $16.67 \pm 4.52 \mathrm{mg} / \mathrm{ml}$ ) and immunoglobulin A (from $377.13 \pm 229.88$ to $484.16 \pm 290.98 \mathrm{ng} / \mathrm{ml}$ ) levels were significantly changed when compared to the baseline value $(p<0.05)$. Conclusion: The results of the current study suggested that the consumption of synbiotics significantly improved subjective and objective parameters involving gut-brain axis in high risk alcoholic patients, and further studies are mandatory to reveal the effects of synbiotics on gut health link to central neurological system.
Access this article online

Website:

http://nepjol.info/index.php/AJMS DOI: 10.3126/ajms.v11i1.26497 E-ISSN: 2091-0576 P-ISSN: 2467-9100

Key words: Synbiotics; Gut-Brain; Alcoholics; AUDIT

\section{Address for Correspondence:}

Dr. Phakkharawat Sittiprapaporn, Assistant Professor and Head, Brain Science and Engineering Innovation Research Group,

School of Anti-Aging and Regenerative Medicine, Mae Fah Luang University, Bangkok 10110, Thailand. Phone: +662 6644361

E-mail: wichian.s@mfu.ac.th (C) Copyright AJMS 


\section{INTRODUCTION}

Among most people, alcohol consumption does not result in dependence or abuse. Nevertheless, a significant group of the population as a whole unavoidably is troubled by chronic alcoholism. Alcohol consumption is the third world ranking of risk factor for morbidity and represents $5.9 \%$ of all deaths worldwide. ${ }^{1}$ Alcohol is involved in a number of diseases, disorders, and injuries, and several social problems..$^{2-4}$

Dysbiosis takes place when lifestyle factors distort the gut ecology of bacteria. ${ }^{5}$ Disturbance to the normal gut flora can also happen when there is an overall overgrowth of bacteria. The 2016 study reported that both acute and chronic alcohol consumption altered specific qualifications of the microbiomecomposition, bacterial overgrowth, and breakdown of the mucosal barrier. ${ }^{6}$ Numerous studies reported that an imbalance of the intestinal microbiota, gut dysbiosis, can lead to many diseases including allergy, ${ }^{7}$ diabetes mellitus, ${ }^{8}$ inflammatory bowel diseases, ${ }^{9-10}$ and obesity as well as results in an elevation in the release of endotoxins, presented by gram-negative and gram-positive bacteria. Endotoxins trigger proteins and immune cells enhancing inflammation. ${ }^{11}$ Although bacterial overgrowth is able to be triggered directly by alcohol, a number of studies report that it may be an indirect byproduct of poor digestive and intestinal function resulted from alcohol consumption.

Collective data demonstrate that the gut microbiome significantly produces the bidirectional communication between the gastrointestinal tract and the brain, which has been known as the microbiota-gut-brain axis. Two vast sources of alcohol related inflammation inducers are alcohol damaged cells and gut microflora, particularly, lipopolysaccharide. Ethanol and their toxic metabolites directly cause the production of reactive oxygen species, known for their mechanism to induce triggering of a main inflammatory transcription factor nuclear factor- $x \mathrm{~B}$ $(\mathrm{NF}-x \mathrm{~B}) \cdot{ }^{12}$ Ethanol induces lipopolysaccharide, a key outer membrane of all Gram-negative bacteria, translocation across the gut though several mechanisms, supported by Fukui et al.'s study showing alcoholic individuals with liver diseases had significantly increased circulating lipopolysaccharide. ${ }^{13}$ Lipopolysaccharide is able to imitate bacterial infection leading to an acute inflammatory response.

After translocating via the gut epithelium, lipopolysaccharide in the interstitial fluid is able to go into the systemic blood circulation by two pathways: the portal vein and the gastrointestinal tract lymphatic vessels. ${ }^{14}$ Then lipopolysaccharide in systemic circulation is reachable to several organs and is involved in multi-organ damage, especially when detoxification process in liver is impaired. Lipopolysaccharide and pro-inflammatory cytokines are able to trigger the neuroendocrine response in the central nervous system resulting in the activation of the hypothalamo-pituitary-adrenal axis and inflammation in central nervous system.

The current study was conducted to evaluate the effect of synbiotics supplement on gut-liver-brain axis in high risk alcoholic patients.

\section{MATERIALS AND METHODS}

\section{Participants}

Twenty-four male participants 20-65 years old with high risk alcohol drinker classified by score of the alcohol use disorders identification test (AUDIT) at 8 or above were participated in this pre- and post-test clinical trial. Exclusion criteria included major abnormal cirrhotic signs, symptoms and laboratory investigations such as jaundice, ascites, asterixis, vomiting blood, hypoalbuminemia, and coagulopathy, history of central nervous system and psychiatric disorders such as epilepsy and brain trauma, human immunodeficiency virus or other immunodeficiency and autoimmune disorder, regular use of drugs other than alcohol, pregnancy and lactation, use of antibiotics during the course of this study, consumption of others dietary supplements and herbs during the course of this study, history of side effects towards pro- or prebiotic supplements. The study was approved by and performed under the guidelines of the Research Ethics Committee of Mae Fah Luang University, Thailand, and a written consent was obtained from all participants. The participants were screened and included into the study according to the inclusion and exclusion criteria. Informed consent was obtained from all study participants before initiating study procedures. Information on demographics and medical history were recorded.

\section{Preparation of synbiotics intervention}

Synbiotics were manufactured by Lactomason Korea Co., Ltd. and contained Lactobacillus rbamnosus, Lactobacillus paracasei, Lactobacillus reuteri, Lactobacillus salivarius, Bifidobacterium lactis, Bifidobacterium breve, Bifidobacterium longum. Total of probiotics contained $6.25 \times 10^{9}$ colony forming unit as well as included Inulin 4 grams, Fructooligosaccharide 2 grams and Galactooligosaccharide 2 grams.

\section{The intervention}

The aluminum foil sachets contained synbiotics were given to the subjects. The subjects consumed one sachet per day for 8 weeks. They were reminded to take it daily by phone 
and allowed to drink normally alcohol but not to ingest the others including others dietary supplements and herbs during the course of this study.

\section{Sample collection}

Blood samples for biochemical tests were collected in a sterile blood collecting tube at baseline and after 8 weeks of intervention and stored on ice or $4^{\circ} \mathrm{C}$ until analysis.

\section{Biochemical analysis}

After the sample collection, biochemical tests included blood Gamma Glutamyl transferase (GGT) (Kinetic photometric method), blood lipopolysaccharide (Mybiosource Human Lipopolysaccharide ELISA kit) and Immunoglobulin A (Elabscience Human IgA ELISA kit) were taken at baseline and end of treatment, $8^{\text {th }}$ week of treatment.

\section{Data analysis}

Descriptive analyses were performed for demographics utilizing characteristic measures such as percent, mean and standard deviation. After using Kolmogorov-Smirnov (KS) test for determining parametric or nonparametric data, analytic analyses used statistical paired sample t-test or Wilcoxon signed rank test for evaluating mean differences

\begin{tabular}{|c|c|c|}
\hline Characteristic & Number (Percentage) & Mean $\pm S D$ \\
\hline Age (years) & & $42.50 \pm 11.66$ \\
\hline Less than 30 & $4(20.0)$ & \\
\hline $30-49$ & $10(50.0)$ & \\
\hline 50 or more & $6(30.0)$ & \\
\hline Weight (kg) & & $62.55 \pm 11.51$ \\
\hline BMI $\left(\mathrm{kg} / \mathrm{m}^{2}\right)$ & & $22.50 \pm 3.36$ \\
\hline Less than 18.5 & $2(10.0)$ & \\
\hline $18.5-22.9$ & $10(50.0)$ & \\
\hline $23.0-24.9$ & $2(10.0)$ & \\
\hline $25.0-29.9$ & $6(30.0)$ & \\
\hline Body fat $(\%)$ & & $16.69 \pm 4.43$ \\
\hline
\end{tabular}

in AUDIT scores and biochemical parameters between baseline and end of treatment ( $8^{\text {th }}$ week). Computer statistical program was used for statistical analyses, and $p$-values of $<0.05$ were considered statistically significant.

\section{RESULTS}

\section{A. Demographic information of the subjects}

Twenty male participants were registered and completed the study. Four participants were dropped out due to loss of follow up. The mean age of the participants was 42.50 \pm 11.66 years. According to Western Pacific regional office of the WHO's Asian BMI cut-off recommendation, about a half of participants are abnormal BMI persons (underweight $10 \%$, Overweight $10 \%$, and Obesity $30 \%$ ) (Table 1). The mean compliance scores calculated from the remaining supplements returned were $90 \pm 6 \%$. Relied on completion of compliance forms, all participants reported to have consumed about $81 \%$ of assigned synbiotics between trials. Analysis of food frequency questionnaire data showed there were no differences in participants consumption habits throughout the intervention period $(p>0.05)$.

\section{B. Changes in alcohol use identification test (AUDIT)} score

There was significantly improved total AUDIT score $(p=0.001)$ as well as the data showed significant decrease in scores of frequency of consuming and blackouts problems from alcohol drinking ( $p=0.011$ and 0.014 respectively). No other differences were observed between trials $(p>0.05)$ (Table 2).

\section{Changes in biochemical levels}

The serum GGT, plasma lipopolysaccharide and immunoglobulin A levels were measured at baseline and after 8 weeks of synbiotic intervention. The level

Table 2: Pre-and Post-Alcohol Use Disorders Identification Test (AUDIT) Scores

\begin{tabular}{|c|c|c|c|c|c|c|c|}
\hline \multirow[t]{2}{*}{ Domains } & \multirow[t]{2}{*}{ Question content } & \multicolumn{2}{|c|}{ Pre-test } & \multicolumn{2}{|c|}{ Post-test } & \multirow[t]{2}{*}{ Mean delta ${ }^{a}$} & \multirow[t]{2}{*}{ p value ${ }^{b}$} \\
\hline & & $n$ & Mean (SD) & $n$ & Mean (SD) & & \\
\hline \multirow[t]{3}{*}{ Hazardous alcohol use } & Frequency of drinking & 20 & $3.35(0.75)$ & 20 & $2.90(0.91)$ & -0.45 & $0.011^{*}$ \\
\hline & Typical quantity & 20 & $2.40(0.75)$ & 20 & $2.45(0.61)$ & +0.05 & 0.564 \\
\hline & Frequency of heavy drinking & 20 & $2.95(0.69)$ & 20 & $2.65(0.75)$ & -0.30 & 0.096 \\
\hline \multirow[t]{3}{*}{ Dependence symptoms } & Impaired control over drinking & 20 & $3.05(0.76)$ & 20 & $2.90(0.79)$ & -0.15 & 0.180 \\
\hline & Increased salience of drinking & 20 & $2.95(0.83)$ & 20 & $2.80(0.70)$ & -0.15 & 0.317 \\
\hline & Morning drinking & 20 & $2.90(0.72)$ & 20 & $2.60(0.75)$ & -0.30 & 0.058 \\
\hline \multirow[t]{4}{*}{ Harmful alcohol use } & Guilt after drinking & 20 & $2.60(0.68)$ & 20 & $2.65(0.67)$ & +0.05 & 0.317 \\
\hline & Blackouts & 20 & $3.05(0.51)$ & 20 & $2.60(0.60)$ & -0.45 & $0.014^{*}$ \\
\hline & Alcohol-related injuries & 20 & $2.20(0.62)$ & 20 & $2.20(0.52)$ & 0 & 1.00 \\
\hline & $\begin{array}{l}\text { Others concerned about } \\
\text { Drinking }\end{array}$ & 20 & $2.20(0.62)$ & 20 & $2.10(0.55)$ & -0.10 & 0.157 \\
\hline Total & & 20 & $27.50(4.07)$ & 20 & $25.65(3.98)$ & -1.85 & $0.001^{*}$ \\
\hline
\end{tabular}

$\mathrm{SD}=$ standard deviation. ${ }^{\mathrm{a}} \mathrm{Calculated}$ using Wilcoxon signed rank test for paired pre-test and post-test data. ${ }^{\mathrm{b}}$ Represents mean of pre-test to post-test score delta for all individual paired data. *indicates the significant difference between samples $(P<0.05)$. 


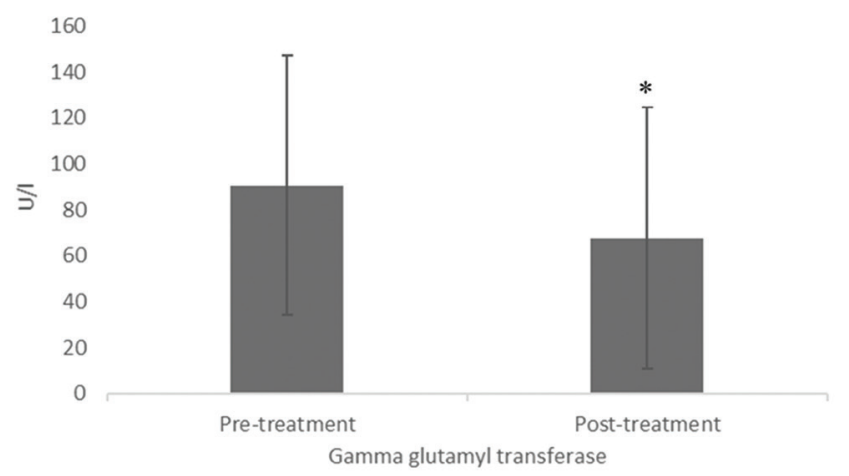

Figure 1: Changes in the level of gamma glutamyl transferase after 8 weeks of synbiotics intervention in high risk alcoholic participants. *indicates the significant difference between samples $(p<0.05)$.

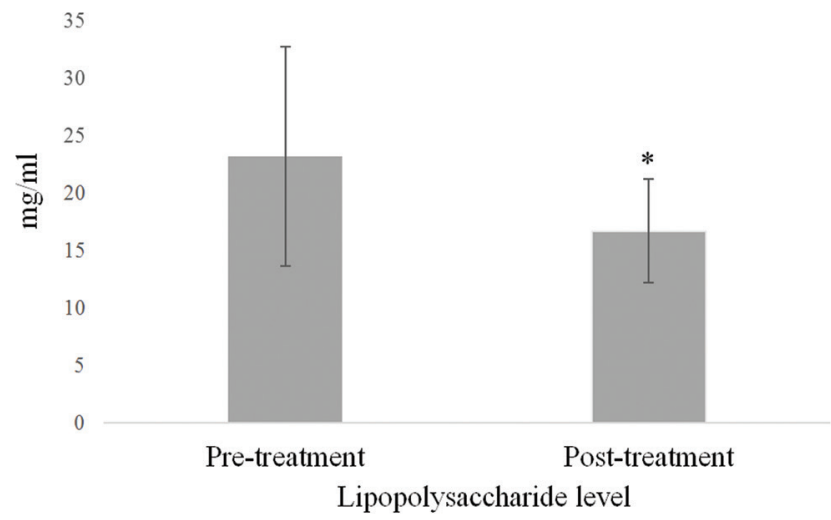

Figure 2: Changes in the level of lipopolysaccharide after 8 weeks of synbiotics intervention in high risk alcoholic participants. *indicates the significant difference between samples $(p<0.05)$.

of GGT, lipopolysaccharide and immunoglobulin $\mathrm{A}$ at baseline (Pre-treatment) were $90.62 \pm 56.65 \mathrm{U} / 1,23.19$ $\pm 9.57 \mathrm{mg} / \mathrm{ml}$ and $377.13 \pm 229.88 \mathrm{ng} / \mathrm{ml}$, respectively. Whilst after 8 weeks of synbiotics supplementation, the level of GGT, lipopolysaccharide and immunoglobulin A were $67.67 \pm 57.00 \mathrm{U} / 1,16.67 \pm 4.52 \mathrm{mg} / \mathrm{ml}$ and $484.16 \pm 290.98 \mathrm{ng} / \mathrm{ml}$, respectively (Figure 1, 2 and 3). After the synbiotics intervention, GGT and lipopolysaccharide level were significantly decreased $(p<0.05)$. whereas immunoglobulin A level was significantly increased $(p<0.05)$.

\section{Adverse events}

Four participants reported experiencing gastrointestinal (GI) symptoms during the study. The most commonly reported GI symptoms were bloating $(n=2)$, and diarrhea $(n$ $=2$ ). No serious adverse events were registered during trials.

\section{DISCUSSION}

Alcohol consumption is able to directly affect several aspects of gut health, such as increased intestinal permeability and

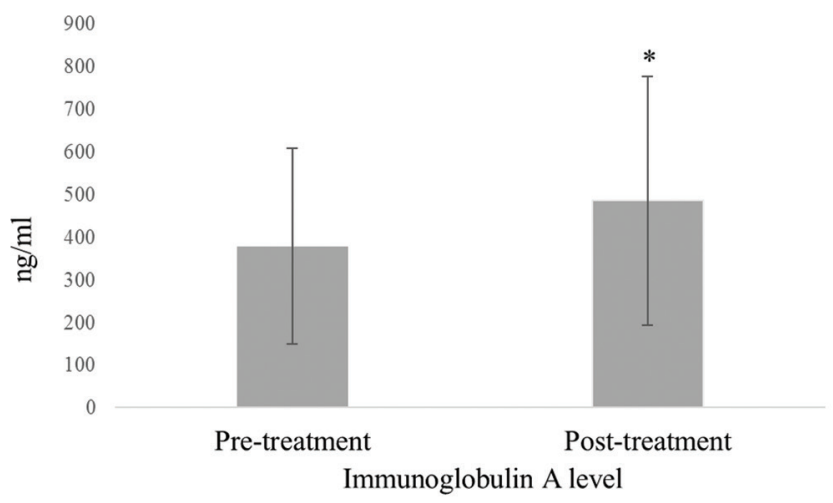

Figure 3: Changes in the level of immunoglobulin A after 8 weeks of synbiotics intervention in high risk alcoholic participants. *indicates the significant difference between samples $(p<0.05)$.

gut dysbiosis, as well as brain functions, including cognitive impairment and increased inhibitory error. Meanwhile, gut and brain provide a communication each other. Key mediators of bidirectional signaling comprise of serotonin (5-HT), opioid, and endocannabinoid, gut hormones, cytokines, and growth factors. ${ }^{15-16}$ Hence, some effects of the one organ can indirectly impact to another one. Several clinical studies show depression and psychological stress are related with exacerbations of inflammatory bowel disease and with the pathogenesis of irritable bowel syndrome. ${ }^{17} \mathrm{It}$ may be described by serotonergic dysregulation and decline of gut barrier function through mast cell-dependent and mast cell-independent mechanisms. ${ }^{18-19}$

The synbiotics administration in alcoholics providing intestinal microbial balance and improving gut health gained much attention in the recent years. According to gut-brain connection, the synbiotics may play a role in amelioration brain functions among chronic alcohol drinkers. Michels et al. showed the effects of probiotics likely associated cognitive control processes further on modulating affective processes and affected prefrontal cortex. ${ }^{20}$ Moreover, several studies supported the positive effects of probiotic supplementation on improving anxiety and depressive symptoms that is considered to stimulate an inclination toward drinking in alcoholic patients. ${ }^{21-22}$ The results of the current study also demonstrated that the synbiotics supplementation decreased subjective alcohol use identification test (AUDIT) score and biochemical parameters (gamma glutamyl transferase and lipopolysaccharide) as well as increased immunoglobulin A levels in high risk alcoholic participants.

\section{CONCLUSION}

The results supported that the consumption of synbiotics contained 6.25 billion cells of probiotics per day for 8 weeks 
significantly improved subjective and objective parameters involving gut-brain axis in high risk alcoholic participants. However, the current study has some downsides including limited sample size, short duration of the study, no-placebo control, vast age differences among the participants $( \pm$ 11.66 years), and limited studied parameters. Thus, a further extended study is required to confirm the results of the present study.

\section{ACKNOWLEDGEMENT}

We thank all of the participants who involved in this study as main data provider. The authors would like to acknowledge the National Research Council of Thailand (Grant No. 126/2561); Postgraduate Studies of Mae Fah Luang University; School of Anti-Aging and Regenerative Medicine, Mae Fah Luang University for the supports, and acknowledge the Innovation Center for Holistic Health, Nutraceuticals and Cosmeceuticals, Faculty of Pharmacy, Chiang Mai University, Thailand, for the necessary provision. Authors also gratefully acknowledge Brain Science and Engineering Innovation Research Group, Mae Fah Luang University grant (2019) (MFU-grant no. 611U109005) and 2020, Thailand for the support.

\section{REFERENCES}

1. World Health Organization, Global Status Report on Alcohol and Health [Online]2014. [Cited 2018 June 26] Available from: www.who.int/substance_abuse/publications/global_alcohol_ report/en.

2. Anderson $P$, Cremona $A$, Paton $A$, Turner $C$ and Wallace $P$. The risk of alcohol. Addiction 1993; 88: 1493-1508.

3. Edwards G, Anderson P, Babor TF, Casswell S, Ferrence R and Geisbrecht N. Alcohol Policy and the Public Good. Oxford University Press, 1994.

4. World Health Organization, "Problems related to alcohol consumption, Report of a WHO Expert Committee," Tech. Report Series. 1980; 650.

5. Belizário $\mathrm{J}$ and Napolitano $\mathrm{M}$. Human microbiomes and their roles in dysbiosis, common diseases, and novel therapeutic approaches. Frontiers in Microbiology 2015; 6: 1050.

6. Leung C, Rivera L, Furness JB and Angus P. The role of the gut microbiota in NAFLD. Nat Rev Gastroenterol Hepatol 2016; 13: $412 \mathrm{e} 25$.

7. Noverr MC and Huffnagle GB. Does the microbiota regulate immune responses outside the gut? Trends Microbiol 2004; 12(12): 562-568.

8. Karlsson FH, Tremaroli V, Nookaew I, Bergström G, Behre C and Fagerberg B. Gut metagenome in European women with normal, impaired and diabetic glucose control. Nature 2013; 498(7452): 99-103.

9. Manichanh C, Rigottier Gois L, Bonnaud E, Gloux K, Pelletier EL and Frangeul $L$. Reduced diversity of faecal microbiota in Crohn's disease revealed by a metagenomic approach. Gut 2006; 55(2): 205-211.

10. Seksik P, Sokol H, Lepage P, Vasquez N, Manichanh $C$ and Mangin I. The role of bacteria in onset and perpetuation of inflammatory bowel disease. Aliment Pharmacol Ther 2006; 24(3): 11-18.

11. Keshavarzian A, Farhadi A, Forsyth CB, Rangan J, Jakate S and Shaikh M. Evidence that chronic alcohol exposure promotes intestinal oxidative stress, intestinal hyperpermeability and endotoxemia prior todevelopment of alcoholic steatohepatitis in rats. Journal of Hepatology 2009; 50(3): 538-547.

12. Gloire G, Legrand-Poels S and Piette J. NF-kappaB activation by reactive oxygen species: fifteen years later. Biochem Pharmacol 2006; 72: 1493-1505.

13. Fukui $\mathrm{H}$, Brauner $\mathrm{B}$, Bode $\mathrm{JC}$ and Bode $\mathrm{C}$. Plasma endotoxin concentrations in patients with alcoholic and non-alcoholic liver disease: reevaluation with an improved chromogenic assay. J Hepatol 1991; 12: 162-169.

14. Azuma $\mathrm{K}$, Akiyama, $\mathrm{M}$, Ebata $\mathrm{T}$, Totsuka $\mathrm{M}$ and Hayasaka $\mathrm{H}$. Endogenous endotoxin absorption and the role of intestinal lymphatics. Jpn J Surg 1983; 13: 535-539.

15. Bravo JA, Forsythe P, Chew MV, Escaravage E, Savignac HM and Dinan TG. Ingestion of Lactobacillus strain regulates emotional behavior and central GABA receptor expression in a mouse via the vagus nerve. Proc Natl Acad Sci USA 2011; 108: 16050-16055.

16. Fetissov $S O$ and Déchelotte P. The new link between gut-brain axis and neuropsychiatric disorders. Curr Opin Clin Nutr Metab Care 2011; 14: 477-482.

17. Mawdsley JE, Macey MG, Feakins RM, Langmead L and Rampton DS. The effect of acute psychologic stress on systemic and rectal mucosal measures of inflammation in ulcerative colitis. Gastroenterology 2006; 131: 410-419.

18. Wallon C, Yang PC, Keita AV, Ericson AC, McKay DM and Sherman PM. Corticotropin-releasing hormone $(\mathrm{CRH})$ regulates macromolecular permeability via mast cells in normal human colonic biopsies in vitro. Gut 2008; 57: 50-58.

19. Demaude J, Levêque $\mathrm{M}$, Chaumaz G, Eutamène H, Fioramonti J and Bueno L. Acute stress increases colonic paracellular permeability in mice through a mast cell-independent mechanism: involvement of pancreatic trypsin. Life Sci 2009; 84: 847-852.

20. Franziska $M$ and Esther $A$. The Gut-Brain Axis: Impact of a Probiotic Intervention on Neurocognitive Measures of Emotion and Cognitive Control. Nijmegen CNS 2013; $12: 53$-78.

21. Meysam P, Joel K, Russell JS and Jennifer C. Probiotic supplementation can positively affect anxiety and depressive symptoms: a systematic review of randomized controlled trials. Nutrition Research 2016; 36: 889 - 898.

22. Petit G, Luminet $O$, Cordovil UM, Monhonval P, Leclercq $S$ and Spilliaert Q. Gender Differences in Affects and Craving in Alcohol-Dependence: A Study During Alcohol Detoxification. Alcohol Clin Exp Res 2017; 41: 421-431. 
Authors Contribution:

VS- Concept and design of the study, manuscript preparation, statistically analyzed; PP- Concept and design of the study, statistically analyzed and

interpreted; PP- Concept and design of the study, statistically analyzed and interpreted; TN- Concept and design of the study, critical revision of the manuscript; CC- Concept and design of the study, critical revision of the manuscript; SS- Critical revision of the manuscript; PS- Concept and design of the study, manuscript preparation, critical revision of the manuscript.

\section{Work attributed to:}

Brain Science and Engineering Innovation Research Group, School of Anti-Aging and Regenerative Medicine, Mae Fah Luang University, Thailand. Innovation Center for Holistic Health, Nutraceuticals and Cosmeceuticals, Faculty of Pharmacy, Chiang Mai University, Thailand;

This research paper is a part of first author's Dissertation in Anti-Aging and Regenerative Medicine, School of Anti-Aging and Regeneration Medicine, Mae Fah Luang University, Bangkok, Thailand.

\section{Orcid ID:}

Dr. Vachrintr Sirisapsombat-iD https://orcid.org/0000-0003-3575-6759

Dr. Parama Pratummas- (D) https://orcid.org/0000-0002-1472-3803

Dr. Phuttharaksa Phumcharoen-10 https://orcid.org/0000-0002-4438-9172

Professor Dr. Thamthiwat Nararatwanchai-10 https://orcid.org/0000-0002-4189-8115

Dr. Chaiyavat Chaiyasut- https://orcid.org/0000-0002-1633-2419

Dr. Sasithorn Sirilun- https://orcid.org/0000-0002-1508-2358

Dr. Phakkharawat Sittiprapaporn- (D) https://orcid.org/0000-0002-4103-9396

Source of Support: National Research Council of Thailand (Grant No. 126/2561); Postgraduate Studies of Mae Fah Luang University; School of Anti-Aging and Regenerative Medicine, Mae Fah Luang University, Thailand; Innovation Center for Holistic Health, Nutraceuticals and Cosmeceuticals, Faculty of Pharmacy, Chiang Mai University, Thailand; Brain Science and Engineering Innovation Research Group, Mae Fah Luang University grant (2019)

(MFU-grant no. 611U109005) and 2020, Thailand, Conflict of Interest: None. 